\title{
QUADRANT ANALYSIS OF PERIPHERAL COLOUR CONTRAST THRESHOLDS CAN BE OF SIGNIFICANT VALUE IN THE INTERPRETATION OF MINOR VISUAL FIELD ALTERATIONS IN GLAUCOMA SUSPECTS
}

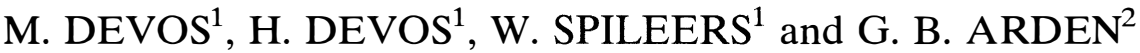 \\ Leuven, Belgium and London
}

\begin{abstract}
SUMMARY
Peripheral colour contrast thresholds were investigated in glaucoma suspects with no or minor visual field alterations, determining the average colour contrast threshold at $12.5^{\circ}$ off axis. The technique was introduced as a rapid screening test for pre-glaucomatous visual loss. Using this 'ring' test, all definite glaucoma patients in the initial study had elevated colour contrast thresholds. In our experience, however, a substantial proportion of early glaucoma patients were found to have normal thresholds. The peripheral colour contrast threshold determined by the ring test is an average threshold in the entire annular zone at $12.5^{\circ}$ eccentricity. We therefore modified the test procedure without a major increase in test duration. An arcade subtending $45^{\circ}$ at $12.5^{\circ}$ eccentricity was used to determine colour contrast thresholds in four quadrants of the visual field separately ('quadrant' test). We present here the first results of this novel procedure, investigating peripheral colour contrast thresholds in glaucomatous patients. The quadrant test is shown to be more sensitive than the ring test in the detection of functional loss.
\end{abstract}

A test for measuring peripheral colour contrast thresholds has previously been described ${ }^{1}$ for the detection of pre-glaucomatous visual loss. An annulus, equiluminous with the background, was - displayed on a high-resolution colour monitor and the colour difference between the stimulus and the

From: ${ }^{1}$ Universitaire Ziekenhuizen, Dienst Oogheelkunde, Leuven, Belgium; ${ }^{2}$ Institute of Ophthalmology, Department of Electrodiagnosis, London, UK.

Correspondence to: W. Spileers, Universitaire Ziekenhuizen, Dienst Oogheelkunde, Kapucijnenvoer 33, B-3000 Leuven, Belgium. background was adjusted to threshold. During the test, $45^{\circ}$ of the annulus was removed in one of four quadrants: upper right, upper left, lower left or lower right. The patient had to identify the correct position of the gap. In this 'four way forced choice' test procedure a modified binary search technique was used to determine the overall colour contrast threshold at $12.5^{\circ}$ off fixation.

We applied this 'ring' test for several years and our results confirmed the loss of peripheral colour contrast sensitivity in glaucoma patients with obvious glaucomatous visual field defects. However, for patients with early glaucomatous visual field loss only $80 \%$ were confirmed by the ring test. When testing the overall peripheral colour contrast threshold, four regions of identical eccentricity are tested at the same time, and the final result 'throws away' the information about the precise retinal locus or loci of malfunction.

It seems likely that averaging the deficits over four quadrants decreases the sensitivity of the test. Therefore we slightly modified the test stimulus to an arcade subtending $45^{\circ}$ at $12.5^{\circ}$ off axis. This stimulus is presented at random in one of four quadrants. The subject has to localise the quadrant in which the arcade is presented. The colour contrast of the stimulus is modulated in each quadrant separately. This 'quadrant' test therefore determines four thresholds, one for each quadrant separately.

We present here the first results of this novel procedure for investigating peripheral colour contrast thresholds in glaucomatous patients. The quadrant test has been shown to be more sensitive than the ring test in the detection of early glaucoma

Eye (1995) 9, 751-756 C 1995 Royal College of Ophthalmologists 
and may be of significant value in the interpretation of minor visual field alterations in glaucoma suspects.

\section{METHODS}

Colour contrast thresholds were measured using the system described in detail by Arden et al. ${ }^{2}$ A personal computer (Compaq Deskpro 386S, 16 $\mathrm{MHz}$ ) displays an image on a high-quality TV monitor (Barco). The display board used is a TSL 206 with a 24 bit palette, operating at $100 \mathrm{MHz}$ on a $980 \times 768$ pixel display with a $94 \mathrm{~Hz}$ refresh rate. The software includes a special calibration program, so that the relations between the bit values set on the $\mathrm{A} /$ D converters of the TSL driver card for the R, G and $B$ guns and the resulting screen luminance are precisely determined. The calibration program also contains values for the CIE standard observer. ${ }^{3}$

To achieve equiluminance between any colour stimulus and the background for each subject the procedure starts with a heterochromatic flicker brightness test. In this test the relative luminance of a red and a green square is adjusted by the subject to achieve minimal flicker sensation. This is repeated for a square flickering between blue and green. All the colours subsequently produced on the screen are adjusted according to the individual relative luminance values of the flicker brightness test to compensate for individual differences in preretinal and retinal absorption. Therefore the stimuli and the background are (psychophysically) precisely equiluminous for each subject.

Colour contrast is defined as the vector distance in the CIE colour space between the colour of the stimulus and the colour of the equiluminous background. The colour contrast threshold is defined as the minimal colour distance between stimulus and background for which the subject is able to differentiate the stimulus from the background, and is expressed as a percentage of the maximum colour contrast arbitrarily defined by the most extreme colours in the colour axis displayed. Colour contrast thresholds were determined along a tritan confusion axis. It was demonstrated ${ }^{4}$ that colour contrast thresholds along these axes are not influenced by the presence of congenital red-green colour defects.

The stimulus proposed by $\mathrm{Yu}$ et al. ${ }^{1}$ was an annulus at $12.5^{\circ}$ off axis where $45^{\circ}$ was randomly removed in one of four quadrants: upper right, upper left, lower left or lower right. The patient had to -identify the quadrant in which the gap was presented, while the colour of the ring was modulated. A 'four way forced choice' modified binary search technique was used to determine the overall peripheral colour contrast threshold. This test will be referred to as the 'ring' test.

In our 'quadrant' test we modified the test stimulus into an arcade subtending $45^{\circ}$ of the annulus. The arcade is presented at random in one of the four quadrants. The patient has to identify in which quadrant the stimulus is presented. Again the 'four way forced choice' modified binary search technique is used. However, the colour of the arcade is modulated for each quadrant individually, and thus colour contrast thresholds are determined in four quadrants separately. The task of the patients was thus changed into a 'positive' stimulus detection; therefore the test was regarded as easier by the great majority of subjects. The increase in test duration is only $20 \%$. This relatively small increase can be explained by the fact that the binary search technique in the quadrant test is run for each quadrant separately and thus has to deal with fewer conflicting responses during the procedure of threshold determination. The test-retest variability was comparable to that of the ring test (see Fig. 2 in Yu et al. ${ }^{1}$ ).

Colour contrast thresholds were determined in normals, glaucoma suspects with no or minor visual field alterations, and glaucoma patients with obvious glaucomatous visual field loss, using both the ring test and the quadrant test.

\section{SUBJECTS}

Forty-one glaucoma suspects, 20 glaucoma patients and 23 normals underwent peripheral colour contrast testing by both ring and quadrant test. All tested subjects were selected from a private ophthalmological practice. Clinical and visual functions were well documented. All had dilated fundus examination with binocular ophthalmoscopy using a +90 dioptre lens. Visual field examinations (program 32 or 34, Octopus 2000) were performed twice a year. For each subject, parameters were obtained from the last visual field by Peridata program 6.2.

We tested 23 eyes of 23 normal subjects, ranging in age from 20 to 66 years. All eyes had a corrected visual acuity of $20 / 20$ or better. All subjects had no known systemic disease and a normal routine ophthalmological examination including an intraocular pressure (IOP) of less than $20 \mathrm{mmHg}$ and a cup/ disc $(\mathrm{C} / \mathrm{D})$ ratio smaller than 0.5 for both eyes. All tested eyes of subjects older than 40 years were tested by automated perimetry and had four reliable visual field examinations without any detectable alteration.

Sixty-one eyes of 61 patients were selected using the following criteria: IOP $>20 \mathrm{mmHg}$ or $\mathrm{C} / \mathrm{D}$ ratio $>0.5$. Twenty eyes were classified as glaucomatous eyes, showing obvious glaucomatous visual field defects on automated perimetry. The mean defects (MD) of the visual fields ranged from $+2.9 \mathrm{~dB}$ to $-11.3 \mathrm{~dB}$, with an average MD of $-6.3 \mathrm{~dB}$. For each subject the regional mean deviation of the quadrant with the most pronounced defect was calculated and 
ranged from $-5.4 \mathrm{~dB}$ to $-22.5 \mathrm{~dB}$, indicating that most glaucomatous eyes showed primarily localised visual field loss. The age in the patient group ranged from 35 to 76 years.

Forty-one patients without obvious glaucomatous visual field defects were regarded as glaucoma suspects. Their age ranged from 44 to 73 years. All eyes had automated perimetry (Octopus 2000, program 32 or 34 ) during an average period of follow-up of 5 years and none of them showed obvious glaucomatous visual field loss. For each subject one eye was chosen at random. The 41 eyes were classified into two groups according to the visual fields. Group I $(n=22)$ showed no visual field alterations on the four previous visual field examinations. The mean defect of the visual fields ranged from $+0.8 \mathrm{~dB}$ to $-4.1 \mathrm{~dB}$, with an average MD of $-1.4 \mathrm{~dB}$. Group II $(n=19)$ had minor visual field alterations (defined as at least one locus of sensitivity loss of $10 \mathrm{~dB}$ or greater, or two adjacent loci of sensitivity loss of at least $5 \mathrm{~dB}$ ), present in the same localisation on the four previous visual field examinations. Alterations were minor and not obviously glaucomatous. In this group the mean defects of the visual fields ranged from $-0.7 \mathrm{~dB}$ to $-8.8 \mathrm{~dB}$, with an average MD of $-3.6 \mathrm{~dB}$.

\section{RESULTS}

\section{Ring Test}

Twenty-three eyes of 23 normal subjects were tested. The mean tritan colour contrast threshold was $14 \%$ $(\mathrm{SD}=4.4)$. These values agree well with earlier published data. ${ }^{1}$

The mean colour contrast threshold on the ring test for glaucoma suspects with normal visual fields (group I) was $19 \%$. There was no significant difference compared with the normals. Six eyes $(27 \%)$, however, had a threshold higher than the normal limit (= mean $+2 \mathrm{SD})$. In glaucoma suspects with minor visual field alterations (group II), the mean colour contrast threshold was $25 \%$ and thus clearly elevated compared with normals. In this group, 11 eyes $(58 \%)$ showed a threshold greater than the normal limit.

For glaucoma patients $(n=20)$ the mean tritan colour contrast threshold was $36 \%$, and ranged from $14 \%$ to $100 \%$. Sixteen eyes $(80 \%)$ showed a peripheral colour contrast threshold on the ring test

Table I. Peripheral colour contrast thresholds

\begin{tabular}{lrrrr}
\hline & \multicolumn{2}{c}{ Glaucoma suspects } & Glaucoma \\
\cline { 3 - 4 } & Normals & \multicolumn{1}{c}{ Group I } & Group II & platients \\
\hline Ring & $13.6 \pm 4.4$ & $19.2 \pm 6.1$ & $25.4 \pm 9.3$ & $35.6 \pm 19.3$ \\
Qmax & $14.7 \pm 3.3$ & $21.2 \pm 8.3$ & $39.0 \pm 21.4$ & $50.5 \pm 26.2$ \\
$\Delta Q$ & $5.1 \pm 2.5$ & $8.4 \pm 6.0$ & $20.0 \pm 17.0$ & $28.4 \pm 22.2$ \\
\hline
\end{tabular}

Values are the mean \pm SD (\%). higher than the normal limit. The values are given in Table I.

Colour contrast thresholds determined by the ring test were statistically higher in glaucoma patients and in glaucoma suspects with minor visual field alterations compared with normals. However, $20 \%$ of the glaucoma patients tested showed normal or borderline values, which is more than $\mathrm{Yu}$ et al. ${ }^{1}$ predicted. In the ring test, peripheral colour contrast is tested in several regions of identical eccentricity at the same time and the final result 'throws away' the information about the precise retinal locus or loci of malfunction. It seems likely that averaging the deficits over four quadrants decreases the sensitivity of the test. Therefore we modified the test stimulus and the same patients were examined with the 'quadrant' test, which determines colour contrast thresholds in each quadrant separately.

\section{Quadrant Test}

On the same day all subjects were also examined with the quadrant test. For each subject colour contrast thresholds were determined in four quadrants separately. The quadrant with the highest threshold is defined as Qmax and the difference between the highest and the lowest quadrant threshold is defined as $\Delta \mathrm{Q}$.

For normals the mean colour contrast threshold in the nasal superior quadrant was $12 \%$, in the temporal superior quadrant the threshold was $14 \%$, in the temporal inferior quadrant it was $12 \%$ and in the nasal inferior quadrant it was $11 \%$. There was a statistical difference only between the thresholds in the temporal superior and nasal inferior quadrants, with slightly higher thresholds in the temporal superior quadrant. The mean Qmax was $15 \%$ $(\mathrm{SD}=3.3)$ and the mean $\Delta \mathrm{Q}$ was $5 \%(\mathrm{SD}=2.5)$. The upper limit of normal is considered to be the mean value plus 2 SD.

For glaucoma suspects with normal visual fields (group I), the mean Qmax was $21 \%$ and the mean $\Delta \mathrm{Q}$ was $8 \%$. The quadrant parameters were slightly higher compared with normals. Of the glaucoma suspects with no visual field alterations, 8 eyes $(36 \%)$ showed an elevated Qmax and 7 eyes (32\%) an elevated $\Delta \mathrm{Q}$ (Fig. 1).

For glaucoma suspects with minor visual field alterations (group II), the mean Qmax was 39\% and the mean $\Delta \mathrm{Q}$ was $20 \%$; thus both Qmax and $\Delta \mathrm{Q}$ were clearly elevated compared with normals. For the glaucoma suspects with minor visual field alterations, 17 eyes (89\%) showed an elevated Qmax and 10 eyes (53\%) showed an elevated $\Delta \mathrm{Q}$.

For glaucoma patients the mean Qmax was 51\% and the mean $\Delta \mathrm{Q}$ was $28 \%$. In this group 19 eyes (95\%) showed an elevated Qmax, and 16 eyes $(80 \%)$ showed an elevated $\Delta \mathrm{Q}$ also. The values of the 

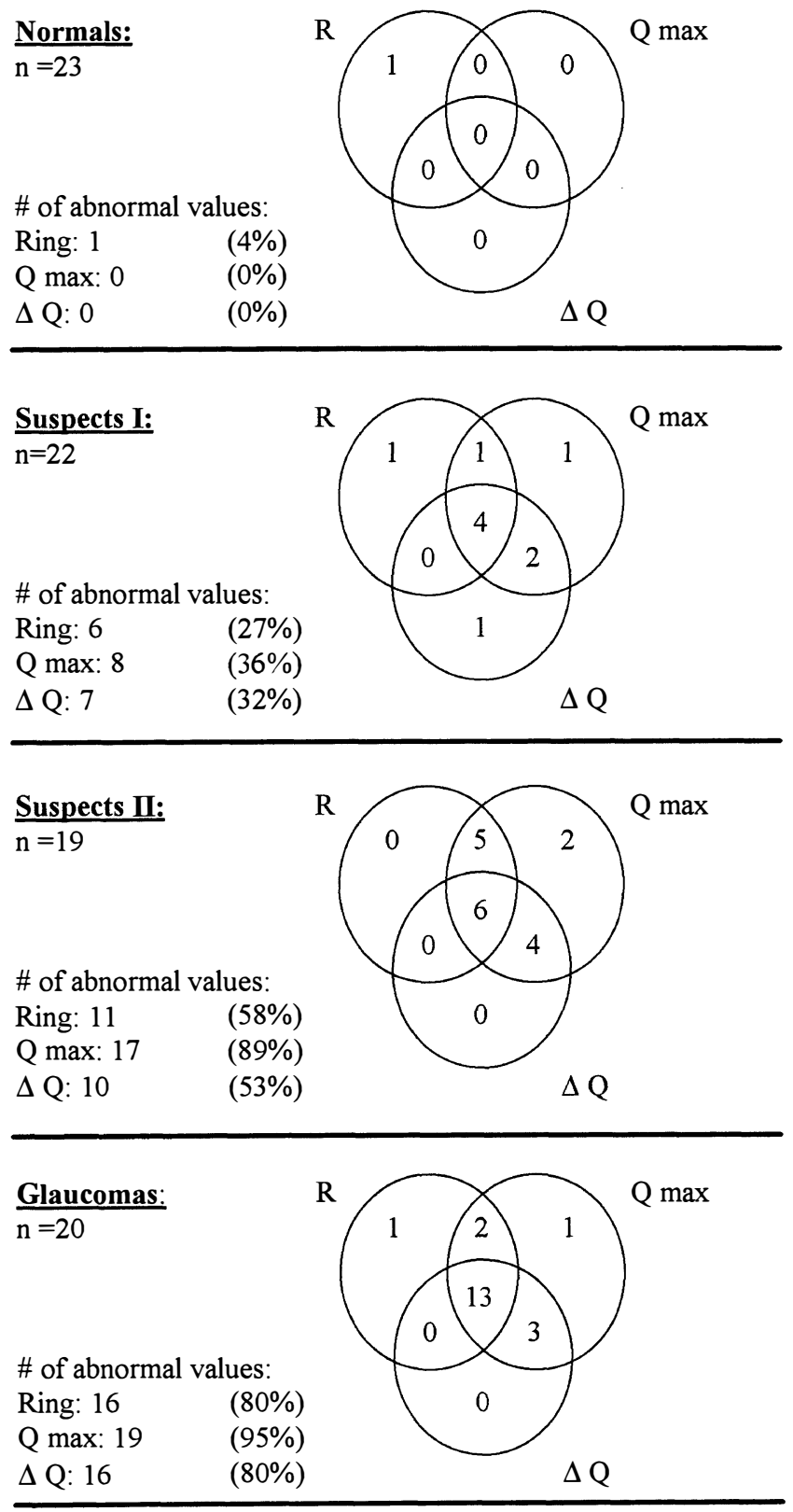

Fig. 1. Venn diagrams illustrating the number of patients with abnormal thresholds on the ring test and abnormal values for Qmax and $\Delta Q$ on the quadrant test and their relation to each other.

different parameters of the quadrant test are given in Table I.

To examine the relationship between the results of the ring test and the different parameters of the quadrant test, a Venn diagram has been constructed showing the number of patients with abnormal values on the different test parameters (Fig. 1). The upper limit of normal is considered to be the mean value plus $2 \mathrm{SD}$ for all parameters, i.e. ring threshold, Qmax and $\Delta \mathrm{Q}$.

\section{Correlation with Visual Field Defects}

Glaucoma Patients. In glaucoma patients showing obvious glaucomatous visual field loss, 4 eyes $(20 \%)$ had normal ring test thresholds. Like Yu et al., ${ }^{1}$ we did not find a good correlation between the degree of the visual field loss scored by the mean deviation and the colour contrast threshold. This can be due to the great variation in the position and degree of localised glaucomatous field loss between persons with equal mean deviation scores. Therefore we analysed the correlation between the localisation of the visual field loss and the localisation of the quadrant with the highest colour contrast threshold (Qmax).

In glaucoma patients we found 19 eyes (95\%) with an elevated Qmax. Sixteen eyes showed a significant inter-quadrant difference in colour contrast threshold (elevated Qmax and elevated $\Delta$ Q: Fig. 1). In 8 eyes the localisation of Qmax corresponded to the quadrant with the most pronounced field defect. In 6 eyes the localisation of Qmax did not correspond with the most pronounced visual field defect, but correlated with glaucomatous alterations in the eccentricity tested by the quadrant test. In 2 eyes Qmax was found in an area with no visual field alterations.

For the 3 eyes with $\Delta \mathrm{Q}$ within normal limits (elevated Qmax but normal $\Delta \mathrm{Q}$ ), 1 eye showed a diffuse depression of the visual field, 1 eye showed no visual field alterations in the eccentricity tested by the quadrant test, and in one eye $(\Delta \mathrm{Q}=9)$ the localisation of Qmax corresponded to the localised visual field defect.

In the group of glaucoma patients 1 eye showed a Qmax and $\Delta \mathrm{Q}$ within normal limits. This eye showed extensive visual field defects in the nasal superior quadrant (regional MD $=-19.5 \mathrm{~dB}$ ), but no visual field alteration in the other quadrant sectors tested.

Glaucoma Suspects with Minor Visual Field Alterations. In glaucoma suspects with minor visual field alterations, 17 eyes $(89 \%)$ were found with an elevated Qmax.

Ten eyes showed a significant inter-quadrant difference in colour contrast threshold (elevated Qmax and elevated $\Delta$ Q: Fig. 1). In all eyes there was a perfect match between the quadrant of the minor visual field alterations and the localisation of Qmax.

Seven eyes showed $\Delta \mathrm{Q}$ within normal limits (elevated Qmax but normal $\Delta Q$ ). In this group 4 eyes showed a match between the localisation of Qmax and the quadrant of the minor visual field alterations. In 3 eyes there was no match between the localisation of Qmax and the visual field alterations; 1 of them showed no visual field alterations in the eccentricity tested by the quadrant test.

In the 2 eyes where Qmax was within normal limits, there were no visual field alterations in the eccentricity tested by the quadrant test. 
Glaucoma Suspects Without Visual Field Alterations. In glaucoma suspects without visual field alterations 8 eyes were found with an elevated Qmax. Six of them showed a significant inter-quadrant difference (elevated Qmax and elevated $\Delta$ Q: Fig. 1). For 1 eye Qmax was localised in the nasal superior quadrant, for 2 eyes Qmax was found in the temporal superior quadrant, for 2 other eyes in the temporal inferior quadrant and for 1 eye in the nasal inferior quadrant. Further follow-up is needed to investigate whether these elevated colour contrast thresholds precede visual field defects.

\section{DISCUSSION}

Peripheral colour contrast thresholds were measured in glaucoma patients and glaucoma suspects with no or minor visual field alterations. The technique, developed by Yu et al., ${ }^{1}$ uses a ring at $12.5^{\circ}$ off axis with a $45^{\circ}$ gap which is randomly presented in one of four quadrants while the colour of the ring is modulated. This concept was introduced to decrease the test duration of a complete field exploration as in automated perimetry. The duration of the ring test is only 3 minutes. However, we found only $80 \%$ of early glaucoma patients to have an elevated threshold on ring test, which was less than predicted by $\mathrm{Yu}$ et al. The colour contrast threshold determined by the ring test is the average colour contrast threshold for the entire annual zone. The procedure 'throws away' the information about the localisation of the colour defects.

In an attempt to increase the sensitivity without a major increase in test duration we modified the test to determine colour contrast thresholds in four different loci at the same eccentricity. An arcade subtending $45^{\circ}$ of the annulus was presented at random in one of the four quadrants. The task of the subjects was 'changed into a 'positive' stimulus detection. The colour contrast of the arcade is modulated in each quadrant separately. The quadrant test thus determines the colour contrast threshold for each quadrant at the specified eccentricity. The quadrant with the highest threshold is defined as Qmax and the difference between the highest and the lowest quadrant threshold is defined as $\Delta \mathrm{Q}$. Because, in the quadrant test, the binary search technique is run for each quadrant independently, the procedure has to deal with fewer conflicting responses in the detection sequence compared with the procedure of the ring test. This explains the only minor increase in test duration of the quadrant test $(20 \%)$.

In glaucoma patients $80 \%$ had an abnormal threshold on the ring test whereas the detection rate was increased to $95 \%$ in the quadrant test. Our results therefore clearly show that the quadrant test is more sensitive than the ring test - without a major increase in test duration.
In glaucoma patients we found no simple correlation between the localisation of the quadrant with the highest colour contrast threshold (Qmax) and the localisation of the most pronounced visual field defect on conventional perimetry. Analysis of all available visual fields by Peridata program 6.2 (at least four examinations for each subject) demonstrates that regions with fluctuating glaucomatous alterations often show higher colour contrast thresholds compared with regions with stable glaucomatous visual field defects. Even normal colour contrast thresholds were sometimes found in sectors with obvious glaucomatous visual field defects. This was the case for the glaucoma patient who had normal colour contrast thresholds on the quadrant test although the visual field showed extensive loss in the nasal superior quadrant (regional $\mathrm{MD}=-19.5$ $\mathrm{dB}$ ). Similar results were found by Felius et al., ${ }^{5}$ who suggested that in some defects the detection in blueon-yellow perimetry is taken over by the luminance channel or by a combination of both the colour channel and the luminance channel. This could explain at least partially the false negative results for colour contrast thresholds in glaucoma patients with obvious but stable visual field defects.

In glaucoma suspects with only minor visual field alterations we did find a good correlation between the localisation of the quadrant with the highest colour contrast threshold (Qmax) and the localisation of the most pronounced visual field alterations. This finding suggests that in glaucoma suspects elevated colour contrast thresholds can help to detect or confirm minor visual field defects. This is in agreement with the results of Johnson et al., ${ }^{6,7}$ who suggest that blue-on-yellow perimetry deficits are predictive of impending glaucomatous visual field loss for standard W/W automated perimetry.

A larger, prospective study has been initiated to define more accurately the relationship between colour contrast thresholds and conventional perimetry in the detection and follow-up of glaucoma patients.

Our preliminary results have shown that measuring colour contrast thresholds in four different quadrants is clearly more sensitive than the overall colour contrast threshold determined by the ring test for the detection of early functional damage in glaucoma. Although only preliminary data are available, our data suggest that the quadrant test described here can be of significant value in the interpretation of minor visual field alterations in glaucoma suspects.

Key words: Glaucoma, Ocular hypertension, Peripheral colour contrast sensitivity, Psychophysics.

\section{REFERENCES}

1. Yu Tak C, Falcao-Reis F, Spileers W, Arden GB. Peripheral colour contrast: a new screening test for pre- 
glaucomatous visual loss. Invest Ophthalmol Vis Sci 1991;32:2779-89.

2. Arden GB, Gunduz K, Perry S. Colour vision testing with a computer graphics system. Clin Vision Sci 1988;2:303-20.

3. Wyszecke G, Stiles WS. Colour science, 2nd ed. New York: Wiley, 1984.

4. Devos M, Spileers W, Arden GB. Colour contrast thresholds in congenital colour defectives. Vision Res 1995;20(3).

5. Felius J, de Jong LAMS, van den Berg TJTP, Greve EL.
Functional characteristics of blue-on-yellow perimetric thresholds in glaucoma. Vision Res 1995;36:1665-74.

6. Johnson CA, Adams AJ, Casson EJ, Brandt JD. Blueon-yellow perimetry can predict the development of glaucomatous visual field loss. Arch Ophthalmol 1993;111:645-50.

7. Johnson CA, Adams AJ, Casson EJ, Brandt JD. Progression of early glaucomatous visual field loss as detected by blue-on-yellow and standard white-on-white automated perimetry. Arch Ophthalmol 1993;111:651-6. 